\title{
Aplicación móvil para acceder a la información académica de la Universidad Francisco de Paula Santander a través de una picocelda bluetooth
}

\author{
Adriana Patricia Wilches Castellanos ${ }^{1} \quad$ Karla Cecilia Puerto López ${ }^{2} \quad \mid$ Jorge Gómez Rojas ${ }^{3}$
}

Recibido:

28 de abril de 2008

Aceptado:

30 de junio de 2009
58

${ }^{1}$ Ingeniera Electrónica. Investigadora. Grupo de Investigación y Desarrollo en

Telecomunicaciones - GIDT. Universidad Francisco de Paula Santander - UFPS. adrianawilches_c@hotmail.com, ${ }^{2}$ Ingeniera Electrónica. Investigadora. Grupo de Investigación y Desarrollo en

Telecomunicaciones - GIDT. Universidad Francisco de Paula Santander - UFPS. karlapuerto16@hotmail.com ${ }^{3}$ Ingeniero Electrónico. Grupo de Investigación en desarrollo Electrónico y Aplicaciones Móviles-GIDEAM. Docente de Planta Universidad del Magdalena. jgomez@unimagdalena.edu.co

\section{Resumen}

Se desarrolló una aplicación móvil celular que permite a los estudiantes de la Universidad Francisco de Paula Santander acceder a parte de la información académica que dicha institución ofrece, aprovechando los beneficios que tiene la tecnología Bluetooth[1]. Se diseño e implemento una picocelda Bluetooth que permite a los usuarios establecer una comunicación mediante un dispositivo móvil celular con tecnología Bluetooth con la fuente de información. Se realizó una evaluación cuantitativa del sistema propuesto en dos condiciones de entornos diferentes, con obstáculos y sin obstáculos, y además se evaluó el potencial de éxito de la aplicación mediante el análisis de las cinco emes (5M's) [2]. Los resultados de la evaluación indican que el sistema es una alternativa viable para acceder a la información sin costo alguno y desde un rango de cobertura establecido.

Palabras clave: Bluetooth, teléfono móvil, JSR-82.

\section{Abstract}

It developed a mobile application that allow to students at the Francisco de Paula Santander University, access to some academic information offered by the Institution, taking advantage of the benefits of Bluetooth technology [1]. A Bluetooth picocell was designed and implemented to allow to users to set a communication between a cellular mobile device with Bluetooth technology enabled and the information source. We performed a quantitative assessment of the proposed system in two different environments: with and without obstacles; and also we assessed the potential for success of implementation through five emes (5M's) analysis [2]. The evaluation results indicate that the system is a viable alternative to offer free access to information within a certain range of coverage.

Keywords: Bluetooth, Mobile phone, JSR-82. 


\section{Introducción}

$\mathrm{E}$ $\mathrm{n}$ respuesta a la necesidad de adquirir información, han surgido nuevas tecnologías que proporcionan medios para acceder a la información en cualquier momento y en cualquier lugar, cada una enfocada en un campo de acción específico [3]. Entre las tecnologías inalámbricas más destacadas se encuentran las de acceso a Redes de Área amplia (Wide Area Network, WAN), el acceso a Redes de Área Local Inalámbrica (Wireless Local Area Network, WLAN), el acceso a Redes de Área Local (Local Area Network, LAN) y el acceso a Redes de Área Personal (Personal Area Network, PAN) regidas por algunos de los estándares 802 del Instituto de Ingenieros Eléctricos y Electrónicos (Institute of Electrical and Electronics Engineers, IEEE) [4]; cada una con un campo de acción diferente, pero que en conjunto conforman una completa solución a los problemas de movilidad; entendiéndose ésta como la capacidad de acceder a la información y los servicios en diferentes zonas de cobertura sin necesidad de una reconfiguración o pérdida de la conectividad a diferencia de portabilidad que solo permite acceder a la información dentro de una zona delimitada[5].

Paralelamente se refleja la aceptación que han generado los dispositivos electrónicos portátiles móviles que permiten almacenar, visualizar e intercambiar información, que ha llevado al éxito de la telefonía móvil y considerando, el impacto que ha supuesto Internet y sus aplicaciones en los sistemas de información, los cuales contribuyen a cambiar los hábitos de la sociedad actual forzando al mercado de las telecomunicaciones a innovar sus tradicionales modelos y conceptos, llegando a un punto en el cual se demanda la convergencia de estos dos campos, para facilitar el acceso a los servicios de información y comunicación desde dispositivos móviles [6].

En la actualidad se han desarrollado aplicaciones en Java2 Micro Edición (Java 2 Micro Edition, J2ME) y diferentes servicios soportados en el Protocolo para Aplicaciones Inalámbricas (Wireless Applications Protocol, WAP) [7] por empresas dedicadas a este rubro exclusivamente, que buscan ofrecer servicios sobre la plataforma de los operadores de telefonía móvil que permitan el intercambio de información entre el dispositivo móvil celular y la fuente de información; ésta información [8] es entregada en paquetes de datos tarifados por la empresa proveedora de servicios, representando costos para el usuario[9].

Además, existen tecnologías alternas no tarifadas, como Bluetooth, para la transferencia de información entre los dispositivos móviles celulares y los servidores de redes locales, como lo es la tecnología Bluetooth [10], y basándose en esta tecnología se desarrolló una aplicación móvil celular que permite acceder a la información académica de la Universidad Francisco de Paula Santander.

Bluetooth es una tecnología inalámbrica que trabaja nivel de radiocomunicación en la banda de frecuencias libres Industrial, Médica y Científica (Industrial, Scientific and Medical, ISM), que comprende rangos de frecuencias reservados internacionalmente para uso no licenciado de radiofrecuencia, como los son 6.7, 13.5, 27.1, 40.6, 433.9 y 915 $\mathrm{MHz}$, y en $2.4,5.8,24.1,61.2,122.5$ y 245 $\mathrm{GHz}$ [11]. El uso de estas bandas está abierto a todo el mundo sin necesidad de concesiones, respetando las regulaciones que limitan los niveles de potencia transmitida, entre otras. Bluetooth, para esta situación, emplea la banda ISM ubicada en los $2.4 \mathrm{GHz}$, lo que hace que esta tecnología sea una alternativa adecuada para la conectividad de dispositivos portátiles móviles.

El objetivo de esta investigación fue establecer una red en configuración ClienteServidor, siendo los Clientes los dispositivos móviles celulares, enlazados por medio de una conexión punto-multipunto basada en la tecnología Bluetooth. 


\section{Materiales y métodos}

Se desarrolló una estructura ClienteServidor basada en la topología de una piconet ${ }^{1}$ en donde el nodo maestro es el Servidor (donde reposa la información académica) y cada uno de los esclavos representa a los Clientes (dispositivos móviles que a través de Bluetooth acceden al nodo maestro), permitiendo llevar a cabo una comunicación bidireccional entre la Universidad Francisco de Paula Santander y sus estudiantes.

Para comenzar con el desarrollo de la aplicación se determinaron las funciones de cada una de las partes de la red y se seleccionaron las herramientas y tecnologías a utilizar para la elaboración de una aplicación que reside en el Servidor de Aplicaciones y una aplicación que reside en el Cliente [12].

El Servidor de Aplicaciones permite gestionar los usuarios de la red y consultar en la base de datos la información solicitada por el Cliente [13]. La aplicación Cliente reside en el dispositivo móvil celular, la cual es enviada por la aplicación Enviar midlet ${ }^{2}$ permitiéndole al estudiante acceder a la información académica que solicite y mostrándola en este dispositivo [14].

\section{A. Arquitectura del sistema}

En la Figura 1, se aprecia la arquitectura general del sistema.

Figura 1. Arquitectura general del sistema.

60

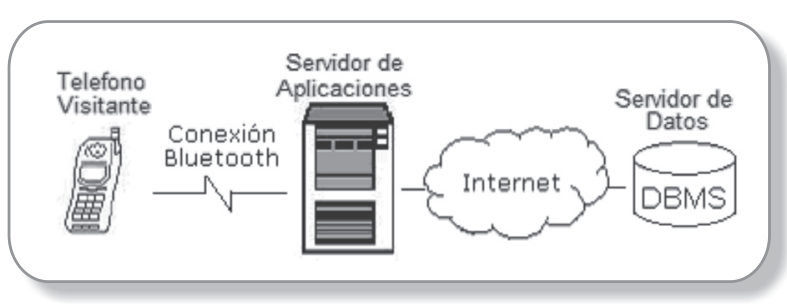

La arquitectura del sistema consta de dos aplicaciones: la aplicación servidora alojada en el Servidor de Aplicaciones, y la aplicación Cliente instalada en el teléfono visitante. Ambas se describen a continuación.

\section{B. Servidor de Aplicaciones}

Un servidor Bluetooth es el encargado de crear una conexión, capaz de especificar los atributos del servicio que se presta en el sistema, y abrir las conexiones con los clientes cuando éstos lo soliciten. El Servidor de Aplicaciones consta de dos tareas una llamada Enviar midlet cuya función principal es descubrir los dispositivos móviles celulares con tecnología Bluetooth dentro del rango de cobertura [15], realiza una búsqueda de servicios en estos dispositivos para determinar si soportan el servicio de intercambio de objetos (OBEX Object Push) y si la búsqueda resulta exitosa establece una conexión con el dispositivo móvil celular y envía el midlet que contiene la aplicación Cliente, luego cierra la conexión; de lo contrario, realiza una nueva búsqueda de dispositivos.

Por otra parte, hay una aplicación llamada Servidor, la cual provee el servicio de información académica y establece una comunicación directa con el dispositivo móvil creando un nuevo servicio llamado Información Académica, permitiendo la transferencia de archivos. Este servicio es único y sólo ofrecido por este equipo Servidor; además, esta aplicación inicializa el dispositivo Bluetooth local para que pueda ser descubierto por otros dispositivos y sea configurado como maestro dentro de la piconet. El dispositivo establece la conexión con un máximo de siete dispositivos al mismo tiempo, proporcionando la información solicitada y cerrando la conexión cuando la comunicación haya finalizado.

\section{Aplicación Cliente}

Es ejecutada en el equipo móvil celular y su función principal es proveer una interfaz gráfica entre el estudiante y la base de datos

\footnotetext{
${ }^{1}$ Conexión de dispositivos Bluetooth que consta de un nodo maestro y hasta siete nodos esclavos activos.

${ }^{2}$ Programa Java para dispositivos embebidos, generalmente son juegos y aplicaciones que funcionan en un teléfono celular.
} 
de la Universidad, para ello cumple con las siguientes tareas: inicializar el dispositivo local, definir su operación como esclavo dentro de la piconet; realizar la búsqueda de dispositivos cercanos y descubrir el servicio Información Académica ofrecido por el equipo Servidor; además, acepta la conexión servidora, envía la información requerida y por último provee una interfaz gráfica que permite visualizar la información consultada.

\section{Herramientas Utilizadas}

El sistema implementado se desarrolló mediante el lenguaje de programación orientado a objetos Java .

La aplicación Servidora se implementó en Java 2 Edición Estándar (Java 2 Standard Edition, J2SE) [2], que es una de las ediciones de Java y es utilizada en computadoras personales. Permite la conectividad con el cliente Bluetooth y el acceso a la base de datos de la Universidad. Se diseñó la presentación de la aplicación móvil celular en Java 2 Micro Edición (Java 2 Micro Edition, J2ME), destinada especialmente a pequeños dispositivos electrónicos de consumo como teléfonos celulares, utilizando la herramienta de desarrollo NetBeans. Además, se diseñó e implementó un programa en Java que descubre los dispositivos Bluetooth cercanos y les envía aplicación cliente desarrollada.

\section{Servidor de Aplicaciones}

El Servidor de Aplicaciones se implementó sobre el sistema operativo Linux, debido a que es distribuido bajo la Licencia Pública General (General Public License, GPL), lo cual significa que puede ser distribuido, copiado y modificado gratuitamente. Se eligió la lista de distribución de Linux Kubuntu 7.04, por su entorno gráfico de fácil manejo, con Kernel 2.6.20 y con soporte Bluetooth a través de la pila oficial de Linux BlueZ.

Las clases implementadas se desarrollaron en J2SE mediante la Interfaz de Programación de Aplicaciones (Application Programming Interface, API) Bluetooth Avetana para Java, la cual es una implementación de código abierto del JSR-82 con la que se puede hacer uso de Bluetooth en aplicaciones para J2SE. Se eligió esta API porque es una versión no comercial para Linux y el uso de esta implementación es transparente para los fines del programador.

\section{Aplicación Cliente}

La aplicación cliente fue diseñada en el Entorno de Desarrollo Integrado NetBeans 5.5.1, siendo el más adecuado para ejecutar aplicaciones en J2ME teniendo en cuenta las herramientas que provee para la edición, compilación, depuración, análisis y ejecución de programas en Java y que incluye las clases del estándar JSR-82 en su paquete de aplicaciones móviles. Además, en este entorno se diseñó la interfaz gráfica del midlet teniendo en cuenta las necesidades y prioridades del usuario, así como las limitaciones técnicas, y el tamaño limitado de la pantalla y memoria que presentan los equipos móviles celulares.

\section{Resultados}

Para evaluar la viabilidad y el correcto funcionamiento del sistema diseñado $\mathrm{e}$ implementado, se evaluó el potencial de éxito de la aplicación móvil mediante el análisis de las 5M's y el desempeño del sistema siguiendo el proceso de una evaluación cuantitativa que permite determinar el comportamiento de la aplicación en diferentes entornos físicos.

\section{A. Evaluación del potencial de éxito}

Para evaluar la aceptación de una aplicación o servicio móvil en el usuario final se aplicó el análisis de las 5M's ).

Tabla1. Evaluación de las 5M's

\begin{tabular}{|c|c|l|}
\hline M & Calificación & \multicolumn{1}{|c|}{ Motivación } \\
\hline $\begin{array}{l}\text { Movimiento } \\
\text { (Movement) }\end{array}$ & 4 & $\begin{array}{l}\text { Se realiza inter- } \\
\text { cambio de infor- } \\
\text { mación indepen- } \\
\text { dien-temente de } \\
\text { la ubicación del } \\
\text { usuario e inde- }\end{array}$ \\
\hline
\end{tabular}




\begin{tabular}{|c|c|c|}
\hline$M$ & Calificación & Motivación \\
\hline & & $\begin{array}{l}\text { pendientemente } \\
\text { de la movilidad } \\
\text { del mismo dentro } \\
\text { de las instalacio- } \\
\text { nes de la UFPS. } \\
\text { El estudiante } \\
\text { no tiene acceso } \\
\text { a los servicios } \\
\text { prestados bajo la } \\
\text { tecnología Blue- } \\
\text { tooth fuera de } \\
\text { la Universidad, } \\
\text { limitando el ser- } \\
\text { vicio a las áreas } \\
\text { de cobertura de } \\
\text { la red Bluetoo- } \\
\text { th dentro de la } \\
\text { UFPS. }\end{array}$ \\
\hline $\begin{array}{l}\text { Momento } \\
\text { (Moment) }\end{array}$ & 4 & $\begin{array}{l}\text { El estudiante } \\
\text { puede consultar } \\
\text { la información } \\
\text { académica en el } \\
\text { momento en que } \\
\text { lo desee mientras } \\
\text { que el servidor } \\
\text { esté activo. } \\
\text { El estudiante } \\
\text { sólo podrá tener } \\
\text { acceso a la infor- } \\
\text { mación académi- } \\
\text { ca a través de la } \\
\text { tecnología Blue- } \\
\text { tooth durante las } \\
\text { jornadas en que } \\
\text { la Universidad } \\
\text { esté abierta a los } \\
\text { estudiantes. }\end{array}$ \\
\hline $\mathrm{Me}(\mathrm{Yo})$ & 5 & $\begin{array}{l}\text { El estudiante re- } \\
\text { cibe en su equipo } \\
\text { móvil celular in- } \\
\text { formación actua- } \\
\text { lizada y persona- } \\
\text { lizada, debido a } \\
\text { que cuando rea- } \\
\text { liza una consulta } \\
\text { sólo podrá acce- } \\
\text { der a informa- } \\
\text { ción relacionada } \\
\text { con las materias } \\
\text { que actualmente }\end{array}$ \\
\hline
\end{tabular}

\begin{tabular}{|c|l|l|}
\hline & & $\begin{array}{l}\text { tenga matricula- } \\
\text { das. } \\
\text { El estudiante po- } \\
\text { drá interactuar } \\
\text { con la base de da- } \\
\text { tos de la Univer- } \\
\text { sidad para resol- } \\
\text { ver sus dudas de } \\
\text { forma personal, } \\
\text { cómoda y priva- } \\
\text { damente. }\end{array}$ \\
\hline Money & 5 & $\begin{array}{l}\text { La aplicación } \\
\text { permite el acceso } \\
\text { a la información } \\
\text { académica de } \\
\text { forma gratuita, lo } \\
\text { que le permite al } \\
\text { estudiante man- } \\
\text { tenerse informa- } \\
\text { do y actualizado } \\
\text { sin ningún costo. } \\
\text { Para la Universi- } \\
\text { dad no represen- } \\
\text { ta ningún costo } \\
\text { ofrecer este ser- } \\
\text { vicio. }\end{array}$ \\
\hline Machines & $\begin{array}{l}\text { La interfaz hom- } \\
\text { bre-máquina del } \\
\text { servicio es sen- } \\
\text { cilla, pero no es } \\
\text { soportada por to- } \\
\text { dos los teléfonos } \\
\text { de los visitantes } \\
\text { debido a las limi- } \\
\text { taciones técnicas } \\
\text { de los dispositi- } \\
\text { vos móviles que } \\
\text { no soportan la } \\
\text { transferencia de } \\
\text { datos mediante la } \\
\text { tecnología. } \\
\text { (Máquinas) }\end{array}$ \\
\hline El crecimiento de \\
la base actual de \\
los usuarios de la \\
telefonía móvil, \\
con teléfono que \\
tengan incorpo- \\
rado la tecnolo- \\
gía Bluetooth. \\
\\
\hline
\end{tabular}


Cada una de las 5M's puede tener un valor entre 0 y 5 , dependiendo que tan bien cumpla el servicio con cada uno de los atributos. Los servicios con alta puntuación en cualquiera de las 5M's se consideran que tienen un gran potencial para el éxito como servicio móvil.

\section{B. Evaluación cuantitativa.}

Para evaluar el desempeño del sistema se diseñó una celda de prueba como la mostrada en la Figura 2. La celda consta de un computador con acceso a Internet mediante una red inalámbrica y con conectividad Bluetooth por medio de un adaptador USB Bluetooth; y de un dispositivo móvil celular que soporta la aplicación Cliente.

Figura 2. Celda de prueba.

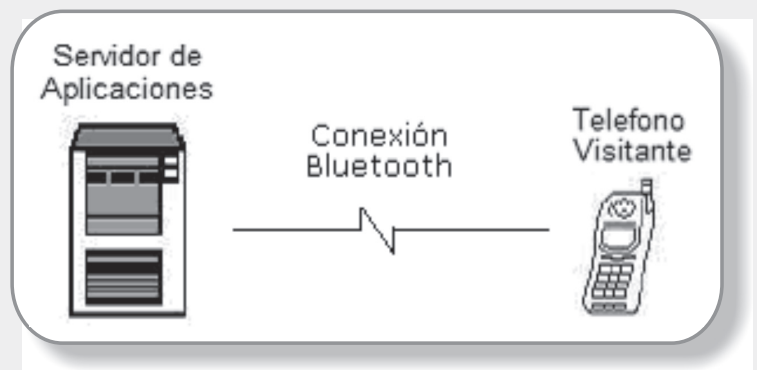

Los dispositivos Bluetooth utilizados son de clase 2, lo que indica que el alcance máximo es de 50 metros, y de acuerdo a las características de los sistemas públicos celulares, el tamaño de la celda corresponde al de una picocelda donde el radio de cobertura es hasta $50 \mathrm{~m}$.

La implementación de la celda de prueba en un entorno sin obstáculos se llevó a cabo en un campo abierto dentro de la UFPS, y la celda de prueba en un entorno con obstáculos fue instalada en el edificio Aulas Generales de la UFPS como se muestra en la Figura 3, en donde la pared que obstaculiza la línea de vista del Servidor de Aplicaciones y el dispositivo móvil celular es de ladrillo (arcilla roja) y de 0.19 metros de grosor.

Figura 3. Celda de prueba con obstáculos.

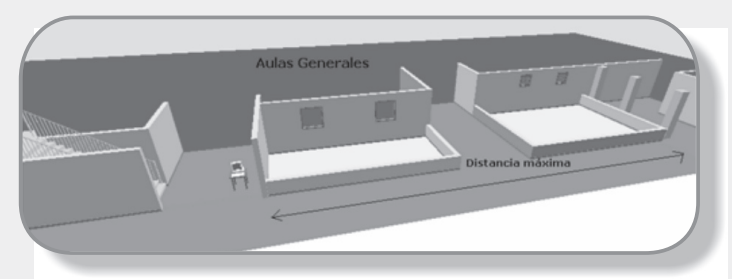

Para esta evaluación cuantitativa se midió el tiempo que tarda el Servidor de Aplicaciones en entregar la información solicitada por la aplicación Cliente en un entorno con presencia de obstáculos y sin ellos. Las mediciones se tomaron cada dos metros de distancia siguiendo el análisis estadístico realizado en el proyecto titulado "Sistema de envío de alertas a terminales móviles basadas en localización utilizando Bluetooth y WAP Push", en donde a cada valor de distancia se toman medidas de tiempo hasta que la desviación estándar es casi constante.

Los resultados de la evaluación cuantitativa sin obstáculos y con obstáculos se muestran en las Tablas 2, 3, 4 y 5.

En esta evaluación se midieron los tiempos de respuesta de la aplicación. Es importante resaltar la presencia de otros dispositivos Bluetooth ajenos a la celda de prueba, además de los factores interferentes que pueden modificar la señal de radiofrecuencia. Los entornos en los que se realizó la evaluación tienen una red WLAN instalada, y las redes Bluetooth y algunas WLAN operan en la misma banda de frecuencia $(2.4 \mathrm{GHz})$ produciendo interferencia la una a la otra [14]. Otro aspecto a tener en cuenta es el tráfico de la LAN de la UFPS, debido a que cuando es alto aumenta el tiempo que tarda el Servidor de Aplicaciones en realizar la consulta en la base de datos, lo que influye directamente en el tiempo de respuesta de la aplicación.

En la evaluación cuantitativa, las medidas de tiempo tomadas no cambian significativamente con la distancia y se puede observar que cuando el dispositivo cliente se encuentra en modo de bajo consumo de energía y se establece la conexión, es decir, pasa a modo activo, el tiempo de respuesta es aproximadamente 10 veces menor que cuando se establece por primera vez la conexión.

Además, se observó que los modelos de los teléfonos móviles celulares no afectan el tiempo de respuesta de la conexión Bluetooth. Esto se afirma partiendo de la similitud de los resultados obtenidos al evaluar la aplicación en los teléfonos de referencia NOKIA 5300 y NOKIA 2760. 
Tabla 2. Análisis estadístico sin obstáculos para el (NOKIA 5300).

\begin{tabular}{|c|c|c|c|c|c|}
\hline $\mathrm{D}(\mathrm{m})$ & $\sigma_{n}$ & $\begin{array}{c}- \\
x \\
(\mathrm{seg})\end{array}$ & $\begin{array}{c}\text { Tmax } \\
(\text { seg })\end{array}$ & $\begin{array}{c}\text { Tmin } \\
(\text { seg })\end{array}$ & $\mathrm{n}$ \\
\hline 0.4 & 0.2757 & $14: 59$ & $15: 01$ & $14: 22$ & 15 \\
5 & 0.1409 & 14.43 & $14: 60$ & $14: 15$ & 15 \\
8 & 0.2732 & $14: 05$ & $14: 53$ & $13: 71$ & 15 \\
10 & 0.3711 & $15: 03$ & $15: 59$ & $14: 58$ & 10 \\
12 & 0.4317 & $14: 92$ & $15: 63$ & $14: 33$ & 10 \\
14 & 0.3722 & $15: 39$ & $16: 01$ & $14: 86$ & 10 \\
16 & 0.5193 & $15: 48$ & $16: 43$ & $14: 74$ & 15 \\
18 & 0.3659 & $15: 53$ & $16: 24$ & $15: 09$ & 10 \\
20 & 0.5115 & $15: 58$ & $17: 05$ & $15: 04$ & 14 \\
22 & 0.4112 & $15: 19$ & $16: 01$ & $14: 39$ & 14 \\
24 & 0.2516 & $14: 85$ & $15: 21$ & $14: 39$ & 10 \\
26 & 0.7295 & $15: 16$ & $16: 19$ & $14: 44$ & 10 \\
28 & 0.7054 & $14: 93$ & $16: 50$ & $14: 17$ & 12 \\
30 & 0.4874 & $16: 01$ & $17: 06$ & $15: 26$ & 15 \\
& & & & & \\
\hline
\end{tabular}

Tabla 3. Análisis estadístico sin obstáculos para el (NOKIA 5300).

\begin{tabular}{|c|c|c|c|c|c|}
\hline $\mathrm{D}(\mathrm{m})$ & $\sigma_{n}$ & $\begin{array}{c}(\bar{x}) \\
(\mathrm{seg})\end{array}$ & $\begin{array}{c}\text { Tmax } \\
(\mathrm{seg})\end{array}$ & $\begin{array}{c}\text { Tmin } \\
(\mathrm{seg})\end{array}$ & $\mathrm{N}$ \\
\hline 0.4 & 0.0751 & $3: 74$ & $3: 89$ & $3: 64$ & 10 \\
5 & 0.1169 & $3: 76$ & $3: 95$ & $3: 59$ & 10 \\
8 & 0.1121 & $3: 38$ & $3: 69$ & $3: 24$ & 15 \\
10 & 0.1588 & $3: 88$ & $4: 11$ & $3: 55$ & 15 \\
12 & 0.1454 & $3: 91$ & $4: 09$ & $3: 58$ & 10 \\
14 & 0.3130 & $3: 86$ & $4: 81$ & $3: 22$ & 15 \\
16 & 0.1496 & $3: 78$ & $3: 93$ & $3: 46$ & 10 \\
18 & 0.2751 & 3.98 & $4: 38$ & $3: 64$ & 20 \\
20 & 0.1867 & $3: 92$ & $4: 29$ & $3: 73$ & 10 \\
22 & 0.3710 & $3: 68$ & $4: 21$ & $3: 14$ & 10 \\
24 & 0.3428 & $3: 61$ & $4: 50$ & $3: 21$ & 15 \\
26 & 0.4107 & $3: 75$ & $4: 60$ & $3: 28$ & 12 \\
28 & 0.3068 & $3: 59$ & $4: 40$ & $3: 30$ & 10 \\
30 & 0.3992 & $3: 91$ & $4: 52$ & $3: 35$ & 18 \\
\hline
\end{tabular}

Tabla 4. Análisis estadístico sin obstáculos para el (NOKIA 2760).
Tabla 5. Análisis estadístico sin obstáculos para el (NOKIA 2760).

\begin{tabular}{|c|c|c|c|c|c|}
\hline $\mathrm{D}(\mathrm{m})$ & $\sigma_{n}$ & $\begin{array}{c}- \\
x \\
(\mathrm{seg})\end{array}$ & $\begin{array}{c}\text { Tmax } \\
(\text { seg) }\end{array}$ & $\begin{array}{c}\text { Tmin } \\
(\text { seg) }\end{array}$ & $\mathrm{N}$ \\
\hline 0.4 & 0.0986 & $3: 84$ & $4: 02$ & $3: 70$ & 15 \\
5 & 0.2337 & $3: 97$ & $4: 36$ & $3: 55$ & 10 \\
8 & 0.2190 & $4: 08$ & $4: 63$ & $3: 74$ & 15 \\
10 & 0.1446 & $3: 93$ & $4: 23$ & $3: 75$ & 12 \\
12 & 0.3162 & $4: 42$ & $5: 11$ & $4: 04$ & 10 \\
14 & 0.5020 & $4: 52$ & $5: 37$ & $3: 70$ & 15 \\
16 & 0.2742 & $4: 25$ & $5: 06$ & $3: 96$ & 12 \\
18 & 0.5367 & 4.57 & $5: 84$ & $4: 14$ & 10 \\
20 & 0.4315 & $4: 58$ & $5: 10$ & $4: 05$ & 10 \\
22 & 0.2636 & $5: 15$ & $5: 73$ & $4: 44$ & 10 \\
24 & 0.1848 & $4: 99$ & $5: 26$ & $4: 57$ & 10 \\
26 & 0.1965 & $5: 00$ & $5: 33$ & $4: 75$ & 10 \\
28 & 0.1672 & $5: 23$ & $5: 52$ & $4: 93$ & 10 \\
30 & 0.3229 & $5: 84$ & $6: 15$ & $4: 99$ & 10 \\
& & & & & \\
\hline
\end{tabular}

\section{Conclusiones}

Con la aplicación móvil implementada se logra acceder a la información académica que proporciona la Universidad Francisco de Paula Santander sin costo alguno y desde un equipo móvil celular con tecnología Bluetooth, ofreciendo a los estudiantes una solución que complementa los servicios proporcionados por la página Web de la institución.

El radio de cobertura de una picocelda Bluetooth se ve directamente afectado por los materiales de construcción de las paredes aledañas, por tanto el mejor escenario para implementar una red de este tipo es un ambiente abierto, sin obstáculos.

Es recomendable orientar el diseño de la picocelda Bluetooth en primera instancia a un análisis de cobertura con un modelo de propagación que actúe en 2,4 Ghz y luego por tráfico ya que se tiene la limitante de solo siete conexiones simultaneas.

Para desarrollar aplicaciones cliente con tecnología Bluetooth como una aplicación móvil, es de gran apoyo el empleo del entorno de desarrollo integrado netbeans que proporciona un conjunto de herramientas que facilitan la construcción de la interfaz gráfica del midlet, provee un entorno de programación que permite editar, compilar y depurar el código Java e incorpora en su 
paquete de aplicaciones móviles las clases recopiladas en el estándar JSR 82.

Ya que el equipo Servidor se encuentra conectado a través de una conexión LAN a la base de datos a consultar es prioritario que exista una conexión troncal de alta velocidad para agilizar la demanda de consultas.

La herramienta de las 5M's permite evaluar el potencial de éxito de una aplicación móvil celular, mostrando que la aplicación móvil diseñada en este proyecto posee un gran potencial de éxito y garantiza la aceptación del servicio de información académica por parte de los estudiantes de la Universidad.

La evaluación Cuantitativa aplicada al sistema implementado determinó que en el diseño de una celda Bluetooth se deben tener en cuenta los obstáculos físicos y el material del cual están construidos presentes debido a que aumentan notoriamente el tiempo de respuesta de los dispositivos Bluetooth que establecen comunicación.

Para implementar la capa de aplicaciones resultó óptimo utilizar el Estándar JSR82 de Avetana puesto que embebe la aplicación diseñada y facilita la comunicación con las capas inferiores de la pila Bluetooth.

\section{Bibliografía}

[1] AALTO, Lauri.; \& GÖTHLIN, N.icklas; \& KORHONEN, Jani. \&; OJALA, Timo. Bluetooth and WAP Push Based Location-Aware Mobile Advertising System, recuperado 22 de junio 2004, http://www.mediateam.oulu. fi/publications/pdf/496.pdf

[2] MORROW, Robert. K. Bluetooth: Operation and Use. Editorial Mc GrawHill. 2002. 567p.

[3] SENDÍN, Alberto. Fundamentos de los Sistemas de Comunicaciones Móviles. MacGraw-Hill, 2004. 627 p.

[4] Sitio oficial del IEEE. Recuperado en 16 de Julio 2004. http://www.ieee.org
[5] BORCHES, P. Pedro D; \& CAMPO, Celeste. Java 2 Micro Edition: Soporte Bluetooth. Universidad Carlos III de Madrid. 2004. 51 p.

[6] PIROUMIAN, Vartan. Wireless J2ME Platform Programming. Editorial Prentice Hall. 2002. 374p.

[7] Sitio oficial de Bluetooth. Recuperado en 15 de Julio 2004. http://spanish. bluetooth.com.

[8] ORTIZ, Enrique. Using the Java APIs for Bluetooth Wireless Technology, Recuperado en 22 diciembre 2004, http://developers.sun.com/mobility/ apis/articles/bluetoothintro/index.html

[9] Perfiles Bluetooth. Recuperado en 26 de Junio 2004. http://grouper.ieee.org/ groups/802/15/Bluetooth/profile_10_ b.pdf

[10] JOYANES, Luis; \& ZAHONERO, Ignacio. Programación Java 2. Algoritmos, estructuras de datos y programación orientada a objetos. Mac Graw Hill,Mc Graw Hill 2002. 711 p.

[11] Ministerio de Comunicaciones de Colombia. Cuadro Nacional de Atribución de Bandas de Frecuencia. Recuperado 10 Julio 2006. http://www. mincomunicaciones.gov.co/Archivos/ Sectorial/CuadroAtribucion.pdf

[12] Programación Java 2. Algoritmos, estructuras de datos y programación orientada a objetos. Mac Graw Hill. 711 p.

[13] PETERSEN, Richard LR. Red Hat Enterprise Linux \& Fedora core 4: The Complete Reference. Editorial Mc Graw-Hill. 2005. 919p.

[14] Página oficial de avetana Recuperado en 15 de Junio 2004. http://www.avetanambh.de/avetana-gmbh/produkte/jsr82. eng.xml.

[15] SANNA, Stefano. Avetana Bluetooth JSR-82 on Linux Ubuntu. Recuperado en 22 de Junio 2004. http://www. gerdavax.it/mobile/avetana-bluetoothjsr-82-on-linux-ubuntu-610/ 